Research Article - Health Sciences

\title{
Global impact and clinical management of severe respiratory syndrome coronavirus-2 (COVID-19)
}

\author{
Alaa Yousef Ghidan ${ }^{1}$, Fatima Yousef Ghethan ${ }^{2}$, Mohammad Ali Alghanmi ${ }^{3}$, Casimeer C Sangeetha ${ }^{4}$, Ernest \\ David $^{5}$, Malik Saleh Haddadin ${ }^{6}$, Mohammad Yasin Mohammad ${ }^{7}$
}

${ }^{1}$ Nanotechnology and Microbiology, Research and Development Center, The Higher Council for Science and Technology, Amman 11941, Jordan

${ }^{2}$ Head of Quality and Medication Safety Unit, King Abdullah Medical City, Al Mashair, Makkah 24246, Makkah 21955 Saudi Arabia

${ }^{3}$ Director of Pharmaceutical Service Administration, , king Abdullah Medical City, Al Mashair, Makkah 24246, Makkah 21955 Saudi Arabia

${ }^{4}$ Department of Physics, Sri Padmavati Mahila Visvavidyalayam (Women University), Andhra Pradesh 517502, India

${ }^{5}$ Department of Biotechnology, Thiruvalluvar University, Vellore 632115, India

${ }^{6}$ Department of Nutrition and Food Technology, University of Jordan, Amman 11910, Jordan

${ }^{7}$ Natural Product and Bioorganic Chemistry, Faculty of Pharmacy, Aqaba University of Technology, Amman 11191, Jordan

(Received: 05-09-2020; Accepted 29-09-2020; Published Online 30-10-2020)

*Corresponding author (E-mail: alaa_ghidan@yahoo.com)

\begin{abstract}
Coronaviruses are enveloped non-segmented positive-sense RNA viruses belonging to the family Coronaviridae and the order Nidovirales and broadly distributed in humans and other mammals. The recommendations for the management of COVID-19 are mentioned in a lot of Updated Literature such as in The "L. Spallanzani" National Institute for the Infectious Diseases. These recommendations are considered as expert's opinions, which may be modified according to newly produced literature data. In addition, Chloroquine and its derivative, hydroxychloroquine, have a long history as safe and inexpensive drugs for use in malaria-endemic regions and as daily treatments for autoimmune diseases, with the most common side effect being eye damage after long-term use. Although previous studies have revealed that chloroquine has therapeutic activity against viruses, including human corona virus OC43 in animal models and SARS-CoV in cell culture studies. we proposed that from natural Medicine the polyphenol compounds in olive leaf extracts were responsible for the stimulation of probiotic Microbes growth and metabolism and that olive leaf extracts ingested in human diet might have the same effect on desirable components of the intestinal microflora, Herbal medicines, plant products, and phototherapeutic have been widely used all over the world since ancient times. Such as Orange, garlic, Bananas, and lemon have an effect on increasing the immune system. On the other hand; Gold nanoparticles (AuNPs) are a piece of evidence to treat the harmful responses arising from reactive oxygen species (ROS). Silver nanoparticles (AgNPs) are microbial agents which could be potentially used as an alternative to anti-viral to treat human infectious disease, especially influenza; there is a novel treatment by using magnetite nanoparticles as nanomedicine drug for Covid- 19.
\end{abstract}

Key words: Medication, Covid-19, Corona virus, Nanomaterials, Phytochemicals, Natural product, Nanocapsulation, Phytotherapeutics

\section{Introduction}

The lockdown has started in late 2019, many researchers started racing to learn more about SARS$\mathrm{CoV}-2$, which is one of a family of viruses known as coronavirus for their crown-like shape (Acter et al., 2020). Northeastern University, Boston, USA, chemical engineer Thomas J. Webster, who specializes in developing nano-scale Medicine and technology to treat diseases, is part of a contingency of scientists that are contributing ideas and technology to the Centers for Disease Control and Prevention to fight the COVID-19 outbreak. The concept of using nanoparticles, Webster says, is that the virus behind COVID-19 mainly constructed of a similar scale as his nanoparticles. At that scale, the matter is ultra-small, about ten thousand times smaller than the width of a single strand of hair( $\mathrm{Hu}$, Frieman, and Wolfram 2020; Liu, Zhou, et al., 2020).
Webster is proposing particles are similar in sizes that could attach to SARS-CoV-2 viruses, concerning their structure with a combination of infrared light treatment that structural change would then sustained the ability of the virus to survive and reproduce in the body. "You have to think in this size range," says Webster, Art Zafiropoulo Chair of chemical engineering at Northeastern. "In the nanoscale size range, if you want to detect viruses, you have to destroy them." Finding and neutralizing viruses with nanomedicine is at the core of what Webster and other researchers call theranostics, which focuses on combining therapy and diagnosis. Using that approach, his lab has specialized in nanoparticles to fight the microbes that cause many diseases like influenza and tuberculosis. "It's not just having one approach to detect whether you have a virus and another approach to use it as a therapy," he says, "but having the same particle, the same approach, for both your detection 
and therapy."

In December 2019, a lot of pneumonia cases of unknown causes emerged in Wuhan, Hubei, China. Following the pneumonia cases were reported in Wuhan and shared history of exposure to sea food stores for all COVID 19 Patient an epidemiological alert and warning was released by the local health authority on Dec 2019, more than 50 -suspected cases with fever and dry cough were transferred to hospitals starting from December 31st, 2019(Zhu et al., 2020). An expert team consists of physicians, Nurses, pharmacist epidemiologists, virologists, and government officials who were soon formed after the alert. Moreover, according to the National Health Commission of China and Ethics Commission of Jin Yin-tan Hospital(Yang et al., 2020). The Ethics Commission of the designated hospital for prominent infectious diseases. In January 2020, the "World Health Organization" (WHO) defined SARS-CoV-2 responsible for the disease (COVID19), some of the cases asymptomatic cases to severe respiratory involvement(Lai et al., 2020). On March 9th, 2020, WHO considers that COVID-19 is a global pandemic(Downing 2020). Besides, Italy isregarded as the second most affected country by COVID-19 infection after China. The "L. Spallanzani" National Institute for the Infectious Diseases, IRCCS, has been the first Italian hospital to admit and manage patients affected by COVID19 (Nicastri et al., 2020). The recommendations for the management of COVID-19, as mentioned in the"L. Spallanzani" National Institute for the Infectious Diseases.These recommendations must be considered as expert opinions, which may be modified according to newly produced literature data(Nicastri et al., 2020).

\section{Treatment and Management}

\section{Pharmaceutical Treatments}

According to National Institute for the Infectious Diseases "L. Spallanzani", IRCCS. Recommendations for COVID-19 clinical management of anti-viral therapy as the following; Remdesivir once per day by intravenous route of Administration the loading Dose $200 \mathrm{mg}$, followed by the maintenance dose of $100 \mathrm{mg}$ daily withinten days, or if Remdesivir isnot available the suggested Alternative Lopinavir/ritonavir* 200/50 mg tablets, two tablets q12h within 28 days (Sun 2020), Also Hydroxychloroquine phosphate $400 \mathrm{mg}$ tablets loading Dose taken as one tablet q12, followed by $200 \mathrm{mg}$ tablets regular Dose 1 tablet q12 withinten days or we can use Chloroquine phosphate instead of Hydroxychloroquine phosphate $250 \mathrm{mg}$ tablets, two tablets q12 within ten days (Capalbo et al., 2020). Before Do not co-administrate Remdesivir with lopinavir/ritonavir, due to possible drug interactions, steroids administration as oral or intravenous rehydration and shall be Considered systemic in case of clinical signs suggesting an incipient worsening of respiratory functions, the steroids are mandatory if Tocilizumab is used with regimen above such as methylprednisolone $1 \mathrm{mg} / \mathrm{Kg}$ daily intravenously for 5 days, followed by $40 \mathrm{mg}$ daily for 3 days and, lastly, $10 \mathrm{mg}$ daily for 2 days, or dexamethasone $20 \mathrm{mg}$ daily intravenously for 5 days, followed by $10 \mathrm{mg}$ daily for 3 days and lastly $5 \mathrm{mg}$ daily for 2 days(Bowen et al., 2012). Also, an article was published regarding the Artificial intelligence predicts that drugs Baricitinib that connected with AP2-related to protein kinase 1 (AAK1) affecting and destroying these, proteins may inhibit viral entry into the target cells, this Medication usually used in the treatment of rheumatoid arthritis, is a kinase inhibitor and indicated for controlling viral replication. Moreover, the remdesivir, also one in vitro clinical study specified that an adenosine analog,which acts as a viral protein inhibitor, has improved the condition in one patient(Zhang et al., 2020).

Chloroquine mechanism of action by rising the endosomal $\mathrm{pH}$ required for virus-cell fusion has the potential of blocking viral infection and was shown to affect activation of p38 mitogen-activated protein kinase (MAPK), which is involved in the replication of HCoV-229E (García et al., 2018). When the patient uses a combination of the antiretroviral drugs lopinavir and ritonavir, it shows significantly improving in the clinical condition of SARSCoV patient(González de Requena et al., 2003).This might be an option in COVID-19 infections. Further possibilities include leronlimab, a humanized monoclonal antibody, and galidesivir, a nucleoside RNA polymerase inhibitor, andthere maybe will be considered as potential treatment candidates(Philippidis 2020). Now almost the Clinical Trials focus on the efficacy of remdesivir immunoglobulins, arbidol hydrochloride oseltamivir, mesenchymal stem cell treatment, hydroxychloroquine, ritonavir plus oseltamivir, lopinavir plus ritonavir methylprednisolone and washed microbiota transplantation (Velavan and Meyer 2020). Another study showed that remdesivir had an excellent therapeutic effect (Wu et al., 2020). Now China has carried out preliminary clinical trials on this drug. Currently, the clinical treatment mainly includes empirical antibacterial drugs, intravenous injection of ribavirin for anti-viral and appropriate Dose of methylprednisolone to relive the shortness of breath U.S (Jin et al., 2020). In addition, there is a suggestion to use traditional Chinese Medicine. This property improves the physical signs of patients, as there was no critical case; no patient used an invasive ventilator, and no death was reported (Wu et al., 2020).

According to recent reports, more than $85 \%$ of patients received anti-viral Medication.For example; oseltamivir at a dose of $75 \mathrm{mg}$ every $12 \mathrm{~h}$ oral route of administration, ganciclovir is given $0.25 \mathrm{~g}$ every $12 \mathrm{~h}$ intravenous route of administration and lopinavir/ritonavir tablets was given with Dose of $400 \mathrm{mg}$ lopinavir and $100 \mathrm{mg}$ ritonavir twice a day orally in combined of the addition of antibiotics that used empirically for $90 \%$ of patients. Even though using intravenous immunoglobulin and systemic steroids have been used in several reports in a particularsituation (Lai et al., 2020; Gorbalenya et al., 2020; Chen et al., 2020; Huang et al., 2020).Also in other reports, several potential drug candidates including anti-viral Medication which include the following lopinavir and ritonavir, tenofovir disoproxil and lamivudine, remdesivir, umifenovir, and chloroquine has been well described, including HIV(Lu 2020) (Wang et al., 2020; Liu, Morse, et al., 2020; Rolain, Colson, and Raoult 2007).

In the current situation, lack of an approved and effective vaccine for severe acute respiratory syndrome coronavirus 2 (SARS-CoV-2), the virus causing COVID-19, it is essential to evaluate potential prophylactic and therapeutic effects of drugs that are clinically approved for other indications (Gao, Tian, and Yang 2020). Chloroquine and its derivative, hydroxychloroquine, have a long history as safe and inexpensive drugs for use in malaria-endemic 
regions and as daily treatments for autoimmune diseases, with the most common side effect being eye damage after long-term use (Kapoor and Kapoor 2020). Although previous studies have revealed that chloroquine has therapeutic activity against viruses, including human coronavirus OC43 in animal models and SARS-CoV in cell culture studies, anti-viral mechanisms of chloroquine remain speculative (Hu, Frieman, and Wolfram 2020; Paraguassu et al., 2020; Salata et al., 2017; SHIQIN 2015). Chloroquine has been used in the field of nanomedicine for the investigation of nanoparticle uptake in cells, and, therefore, insights from synthetic nanoparticle interactions with cells in the presence of chloroquine may reveal mechanisms that are active at early stages before viral replication. Specifically, nanomedicine studies may provide clues on chloroquine-induced alterations of SARS-CoV-2 cellular uptake (Hu, Frieman, and Wolfram 2020)

\section{Probiotics Treatment}

The best supplement to improve the immune system is to take probiotics and prebiotic about one capsule after breakfast.This type of supplement will produce compounds called immunomodulators to supply the immune system cells (Oyetayo and Oyetayo 2005).These compounds work to enhance the immune system efficiency against pathogens like viruses. It is a supplement that contains microbiota: Lactobacillus acidophilus and other species of Lactobacillus with Bifidum species. They will be located on colon tissues. This treatment not affected directly but enhances the immune system to attack the viruses like anyexternal bodies inside the human body(Candela et al., 2008).

Olive leaves implicate high quantities of phenol substances very similar to those present in olives and their derived products(Ghidan et al., 2018). There is compelling scientific evidence that olive leaf polyphenols are bioactive compounds. Olive leaves or their specific organic constituents has significant antimicrobial(Bisignano et al., 1999), antioxidant(Ruiz-Gutiérrez et al., 1999) and antiinflammatory properties(Briante et al., 2002), atherosclerosis inhibition and hypotensive action(Lee et al., 2009; Omar 2010) and anticarcinogenic properties that lead to the prevention of some cancers(Owen et al., 2004). Beneficial properties of olive leaf extracts are further consolidated by the bioavailability of their polyphenolic constituents, which are readily absorbed through the gastrointestinal tract, resulting in significant levels in the circulation system(Visioli et al., 2000; Vissers et al., 2002).

In relation to humans, much concernshave been focused on phenolic compounds from plants and foods that may adjust microbiota in the intestine by selectively increasing that growth of and lactobacilli and decreasing that harmful bacteria such as clostridia(Haddadin 2010). The olive leaf polyphenol composition is alike to that of olive oil. Oleuropein and other secoiridoids are the concept combination while simple phenols, enclosed hydroxytyrosol, are shown but in lower amounts (Tuck and Hayball 2002). Olive leaves consist of flaronoids such as: rutinflavonol, Luteolin-7-glucoside(Pereira et al., 2007).The olive leave extract was shown to have an antioxidant capacity $400 \%$ higher than vitamin $\mathrm{C}$ and almost double that of green tea or grape seed extracts (Ryan, Robards, and Lavee 1999).

As asignificance of the high incidence of civilization, diseases, Science, and industry direct their interests toward the production of food products, which are beyond the normal nutritional function, deliver health benefits. These products are called functional foods, and the probiotics are an example(Fuller 1991; Roberfroid 2000). Recently, the developed countries have arrived at a new food formulation, originated from the combination of nutraceutical compounds and probiotic microorganisms, and formulations are mounting quite a trend (De Leonardis et al., 2008).The growing popularity of functional food causes increasing interest in raw materials, which can raise the value of food when supplemented (Duda-Chodak, Tarko, and Statek 2008). Different results were obtained regarding the effects of plant extracts rich in polyphenolic compounds on the growth of probiotic bacteria and other microorganisms. It was proved that plant extracts could inhibit the growth of food associated with pathogens and microbes.

\section{Nanotechnology Treatments}

Nonomaterial's (N.M.s) have unique physicochemical properties in providing versatile scaffolds for functionalization with biomolecules. Moreover, certain N.M.s such as gold and magnetic nanoparticles as well as polymeric or hybrid N.M.s have shown a respond to external stimuli achieving a spatiotemporal controlled release of macromolecules (Mout et al., 2012; Sapsford et al., 2013). For these reasons, over the last two decades, engineered nanomaterials have been successfully tested and applied in Medicine and pharmacology. Gold nanoparticles (AuNPs) are a piece of evidence to treat the harmful responses arising from reactive oxygen species (ROS). Silver nanoparticles (AgNPs) are microbial agents which could be potentially used as an alternative to anti-viral to treat human infectious disease, especially influenza. Virus infections were unsuccessfully treated by anti-viral (Ghidan et al., 2017; Al and Ghidan, 2018; Mariadoss et al., 2019).

However, many concerns about the use of AgNPs on humans arise from their potential toxicity, although mechanisms are not well-understood. We represent here, in the context of influenza virus infection of lung epithelial cells, that AgNPs down-regulated influenza induced CCL-5 and -IFN- $\beta$ release (two cytokines important in anti-viral immunity) through RIG-I inhibition, while enhancing IL-8 production, a cytokine important for mobilizing host antibacterial responses (Rezvani et al., 2019). AgNPs activity was independent of coating, and it was not observed with gold nanoparticles.

Down-stream analysis indicated that AgNPs disorganized the mitochondrial network. It prevented the anti-viral IRF-7 transcription factor influx into the nucleus. Importantly, we represent that the modulation of the RIG-I-IRF-7 pathway was concomitant with inhibition of either classical or alternative autophagy (ATG-5- and Rab-9 dependent) depending on the epithelial cell type used (Villeret et al., 2018). Altogether, this demonstration of AgNPs-mediated functional dichotomy (down-regulation of IFN-dependent anti-viral responses and up-regulation of IL-8-dependent antibacterial responses) may have practical implications for their use in the clinic (Villeret et al., 2018). Chloroquine, an approved malaria drug, is known in 
nanomedicine research for the investigation of nanoparticle uptake in cells, and it may have the potential for the treatment of COVID-19 (Shukla et al., 2020). Gold, silver, zinc, and copper nanoparticles can be affected by this virus, as well as Magnetite nanoparticles (Patient as nano capsulation).

\section{Natural products and biochemicals}

Eclipta prostrata and known for its therapeutic values in the cure of viral infections, human bronchial epithelial cell injury. It has many pharmacological properties such as anti-inflammatory, anti-cancer, and antioxidant activities(Feng et al., 2019; Li and Kan 2017).

\section{Immunomodulatory activity of some monoterpenes limonene and carvone}

The action of the immunomodulatory activity of some monoterpenes was studied on animals like miceadministration of carvone and limonene like other various monoterpenes. The researches indicate the increase of the total count of white blood cells (WBC) in mice. The maximum total of WBC count in carvone treated mice appeared on the 12th day while in limonene was on the 9th day after the combined administration of carvone and limonene increased the total antibody production. Antibody producing cells in the spleen, bone marrow cellularity (figure 1), and alpha-esterase positive cells significantly compared to the healthy animals indicating its potentiating effect on the immune system (De Carvalho and Da Fonseca 2006; Andrade and De Sousa 2013; Kalsum et al.,, 2017).<smiles>C=C(C)C1CC=C(C)C(=O)C1</smiles>

Carvone

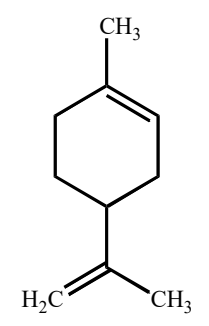

Limonene
Figure 1: Naturally occurring monoteroenes carvone and limonene

\section{Food and Some Nutritional Elements}

Herbal medicines, plant products, and phototherapeutic have been widely used all over the world since ancient times. Such as Orange, garlic, Bananas,and lemon have an effect on increasing the immune system (Vanderhaeghe 2000). Anti-viral properties of garlic were shown in vitro activity against influenza A and B (Weber et al. 1992). Regarding orange, there is much literature regarding orange extracts the results of the provided anti-viral activity. These properties suggest that this orange peel could provide advantages as a topical prophylactic/therapeutic agent for some viral infection (Guo et al., 2012; Marzouk 2013; Huang et al., 2015).

\section{Prevention and Disinfectants}

The most efficient solution to destroy the coronavirus is a solution contains the following compounds: $71 \%$ ethanol, $1 \%$ hydrogen peroxide and $0.10 \%$ Sodium hypo chlorate by using as external prevention for cleaning and disinfectant (Pradhan et al., 2020)

\section{Novel treatments}

SARS-CoV-2 escalates mostly through tiny droplets of viral particles from breathing, talking, sneezing, coughingthat enter the body through the eyes, mouth, and nose. Prospective role of inanimate surfaces for the spread of coronaviruses and their distraction with disinfectant agents. It suggests that those germs may survive for days when they attach themselves to countertops, handrails, and other hard surfaces (Kingsbury et al. 2020). That's one reason to make theranostics with nanoparticles the focus of the COVID-19 outbreak. Nanoparticles can damage these pathogens even before they break into the body, as they hold on to different objects and surfaces(Albrecht, Evans, and Raston 2006). His lab has developed materials that can be spattered on objects to form nanoparticles and attack viruses.

"Even if it was on a surface, on someone's countertop, or a mobilephone," he says. "It doesn't mean anything because it's not the active form of that virus.That same technology can be fine-tuned and changed to target a wide range of viruses, bacteria, and other pathogens. Unlike other novel drugs with large molecular structures, nanoparticles are so small that they can move through our body without disrupting other functions, such as those of the immune system.

"Almost like a surveyor, they can go around your bloodstream," Webster says. "They can survey your body much easier and under much longer times and try and detect viruses." To do all that, the Centers for Disease Control and Prevention (CDC) need to know the specifics about what kind of structure is required to neutralize SARS-CoV-2, Webster says.

An alternative to nanomedicine is producing synthetic molecules. But Webster says that tactic presents some challenges(Alvarez et al., 2017). In the case of chemotherapies used to treat cancer cells, such synthetic drugs can cause severe side effects that kill cancer cells, as well as other cells in the body (Sak 2012). The same thing could be happening with synthetic chemistry to treat a virus, where molecules are killing a lot more than just that virus (Wimmer et al., 2009). Webster says. Still, Webster acknowledges that there aren't many researchers focusing on nanoparticles to kill microorganisms. One of the main reasons for the lack of those solutions is that the same benefits that make nanoparticles ideal to fight infectious diseases also make them a concern for the U.S. Federal Drug Administration. Because of their size, nanoparticles are extensive to seep through other parts of the body (Buzea, Pacheco, and Robbie 2007; Ratner and Ratner 2004). To decrease that risk, Webster's lab has focused on using iron oxide. Particles of that makeup entail chemistry that is already natural to our bodies and diets.

Even if you have a viral infection, you need more iron, because you could be anemic depending on how bad the infection is. "We're developing these nanoparticles out of chemistries that can help your health. Iron-based nanoparticles could be directed with magnetic fields to target specific organs in the body, such as lungs and other areas susceptible to respiratory complications after contracting viral infections (Ratner and Ratner 2004; Greish et al., 
2018). That too, Webster says, is something that you couldn't do with a novel synthetic molecule. What this all means is that we just have to do the studies to show those iron nanoparticles are not going into the brain or the kidney and that these nanoparticles are going exactly where you want them to go to the virus.

\section{Expected Medication to treat corona virus}

\section{First family:Antimalaria}

\section{Chloroquine:}

It used for the treatment of acute malaria. As chemoprophylaxis also the mod of action (MOA) binds to and inhibits DNA and RNA polymerase interferes with metabolism and hemoglobin utilization by parasites inhibits prostaglandin effects, chloroquine concentrates within parasite acid vesicles. It raises internal $\mathrm{pH}$ resulting in inhibition of parasite growth, andit may involve aggregates of ferriprotoporphyrin IX acting as chloroquine receptors causing membrane damage that may also interfere with nucleoprotein synthesis (Wellems and Plowe 2001; Krogstad et al., 1987; Trape et al. 1998). The proposed mechanism involves chloroquine-induced suppression of PICALM, which prevents endocytosis-mediated uptake of SARS-CoV2 '5

\section{Quinine}

It was conjunction with other antimalaria, and for uncomplicated chloroquine-resistant P. falciparum malaria, MOA depresses oxygen uptake and carbohydrate metabolism, intercalates into DNA disrupting the replication and transcription of the parasites, cardiovascular effects similar to quinidine(Krishna and White 1996; Denes, Gabster, and Huang 1981).

\section{Second family:Antiviral}

\section{Amantadine}

As an anti-viral agent and anti-Parkinson's agent, the MOA as anti-viral blocks the uncoating of influenza A virus, preventing the penetration of the virus into host.As anti Parkinsoninactivity may be due to it's blocking the reuptake of dopamine into presynaptic neurons or by increasing dopamine release from presynaptic fibers(Davies et al., 1964; Staničova, Miškovsky, and Šutiak 2001).

\section{Rimantadine}

Prophylaxis adult and children $>1$ and treatment for an adult of influenza A viral infection (45), the MOA exerts its inhibitory effect on three antigenic subtypes of influenza a virus (H1N1, H2N2, H3N2 ) early in the viral explicative cycle possibly inhibiting the uncoating process it has no activity against influenza $B$ virus, 2-8fold more active than amantadine(Tominack and Hayden 1987; Jefferson et al., 2002). On the other hand, the researchers found that the molecular bond between SARS-CoV-2's spike protein and ACE2 looks fairly similar to the binding pattern of the coronavirus that caused the outbreak of SARS in 2003(McNamara, Richt, and Glickman 2020). There are some differences, and however, in the precise amino acids used to bind SARS-CoV-2 to that ACE2 receptor compared with the virus that causes SARS (severe acute respiratory syndrome), this Medication maybe can use to treat coved 19 (Denis et al., ; Prabakaran, Xiao, and Dimitrov 2004).

\section{Conclusion}

Chloroquine and its derivative, hydroxychloroquine, have a long history as safe and inexpensive drugs for use in malaria-endemic regions and as daily treatments for autoimmune diseases, with the most common side effect being eye damage after long-term use (Kapoor and Kapoor 2020). Although previous studies have revealed that chloroquine has therapeutic activity against viruses, including human coronavirus OC43 in animal models and SARS-CoV in cell culture studies, Herbal medicines, plant products, and phototherapeutic have been widely used all over the world since ancient times. Such as Orange, garlic, Bananas, and lemon have an effect on increasing the immune system. Silver nanoparticles (AgNPs) are microbial agents which could be potentially used as an alternative to anti-viral to treat human infectious disease, especially influenza; there is a novel treatment by using magnetite nanoparticles as nanomedicine drug for Covid- 19.

\section{Acknowledgments}

We acknowledge The Higher Council for Science and Technology, Research and Development Center, King Abdullah Medical City Saudi Arabia, as well as Department of Biotechnology, Thiruvalluvar University, Tamilnadu, India, Royal Scientific Society also The Jordan University. Our Special acknowledgement to Jannat Yousef Ali Ghidan (Master of Education), EFL Teacher at Tazkeya Academy to edit this manuscript.

Competing interests: The authors declare no competing interests.

\section{References}

Acter, Thamina, Nizam Uddin, Jagotamoy Das, Afroza Akhter, Tasrina Rabia Choudhury, and Sunghwan Kim. 2020. 'Evolution of severe acute respiratory syndrome coronavirus 2 (SARS-CoV-2) as coronavirus disease 2019 (COVID-19) pandemic: A global health emergency', Science of the Total Environment: 138996.

Albrecht, Matthew A, Cameron W Evans, and Colin L Raston. 2006. 'Green chemistry and the health implications of nanoparticles', Green chemistry, 8: 41732.

Alvarez, Mario M, Joanna Aizenberg, Mostafa Analoui, Anne M Andrews, Gili Bisker, Edward S Boyden, Roger D Kamm, Jeffrey M Karp, David J Mooney, and Rahmi Oklu. 2017. "Emerging trends in micro-and nano-scale technologies in medicine: From basic discoveries to translation." In.: ACS Publications.

Andrade, Luciana Nalone, and Damião Pergentino De Sousa. 2013. 'A review on anti-inflammatory activity of monoterpenes', Molecules, 18: 1227-54.

Bisignano, Giuseppe, Antonio Tomaino, Rossella Lo Cascio, Giuseppe Crisafi, Nicola Uccella, and Antonella Saija. 1999. 'On the in-vitro antimicrobial activity of oleuropein and hydroxytyrosol', Journal of pharmacy and pharmacology, 51: 971-74.

Bowen, Michael, Herren Wu, William Dall'Acqua, Peter A Kiener, Bahija Jallal, and Anthony Coyle. 2012. "Human Anti-IL-6 Antibodies With Extended In Vivo Half-Life And Their Use In Treatment Of Oncology, Autoimmune 
Diseases And Inflammatory Diseases." In.: Google Patents.

Briante, Raffaella, Maurizio Patumi, Stefano Terenziani, Ettore Bismuto, Ferdinando Febbraio, and Roberto Nucci. 2002. 'Olea europaea L. leaf extract and derivatives: antioxidant properties', Journal of Agricultural and Food Chemistry, 50: 4934-40.

Buzea, Cristina, Ivan I Pacheco, and Kevin Robbie. 2007. 'Nanomaterials and nanoparticles: sources and toxicity', Biointerphases, 2: MR17-MR71.

Candela, M, F Perna, P Carnevali, B Vitali, R Ciati, P Gionchetti, F Rizzello, M Campieri, and P Brigidi. 2008. 'Interaction of probiotic Lactobacillus and Bifidobacterium strains with human intestinal epithelial cells: adhesion properties, competition against enteropathogens and modulation of IL-8 production', International journal of food microbiology, 125: 286-92.

Capalbo, Carlo, Antonio Aceti, Maurizio Simmaco, Rita Bonfini, Monica Rocco, Alberto Ricci, Christian Napoli, Matteo Rocco, Valeria Alfonsi, and Antonella Teggi. 2020. 'The Exponential Phase of the Covid-19 Pandemic in Central Italy: An Integrated Care Pathway', International Journal of Environmental Research and Public Health, 17: 3792.

Chen, Nanshan, Min Zhou, Xuan Dong, Jieming Qu, Fengyun Gong, Yang Han, Yang Qiu, Jingli Wang, Ying Liu, and Yuan Wei. 2020. 'Epidemiological and clinical characteristics of 99 cases of 2019 novel coronavirus pneumonia in Wuhan, China: a descriptive study', The Lancet, 395: 507-13.

Davies, WL, RR Grunert, RF Haff, JW McGahen, EM Neumayer, M Paulshock, JC Watts, TR Wood, EC Hermann, and CE Hoffmann. 1964. 'Antiviral activity of 1-adamantanamine (amantadine)', Science, 144: 862-63.

De Carvalho, Carla CCR, and M Manuela R Da Fonseca. 2006. 'Carvone: Why and how should one bother to produce this terpene', Food Chemistry, 95: 413-22.

De Leonardis, Antonella, Alessandra Aretini, Gabriele Alfano, Vincenzo Macciola, and Giancarlo Ranalli. 2008. 'Isolation of a hydroxytyrosol-rich extract from olive leaves (Olea Europaea L.) and evaluation of its antioxidant properties and bioactivity', European Food Research and Technology, 226: 653-59.

Denes, Pablo, Alan Gabster, and Shoei K Huang. 1981. 'Clinical, electrocardiographic and follow-up observations in patients having ventricular fibrillation during holter monitoring: Role of quinidine therapy', The American journal of cardiology, 48: 9-16.

Denis, Martine, Valerie Vandeweerd, Rein VERBEEKE, Anne Laudisoit, Tristan REID, Emma HOBBS, and Laure WYNANTS. 'Information available to support the development of medical countermeasures and interventions against COVID-19'

Downing, SL. 2020. 'COVID-19: a global pandemic', South African Pharmaceutical Journal, 87: 31-34.

Duda-Chodak, Aleksandra, Tomasz Tarko, and Mateusz Statek. 2008. 'The effect of antioxidants on Lactobacillus casei cultures', Acta Scientiarum Polonorum Technologia
Alimentaria, 7: 39-51.

Feng, Li, Yuan-Yuan Zhai, Jia Xu, Wei-Feng Yao, Yu-Dan Cao, Fang-Fang Cheng, Bei-Hua Bao, and Li Zhang. 2019. 'A review on traditional uses, phytochemistry and pharmacology of Eclipta prostrata (L.) L', Journal of ethnopharmacology: 112109.

Fuller, Roy. 1991. 'Probiotics in human medicine', Gut, 32: 439.

Gao, Jianjun, Zhenxue Tian, and Xu Yang. 2020. 'Breakthrough: Chloroquine phosphate has shown apparent efficacy in treatment of COVID-19 associated pneumonia in clinical studies', Bioscience trends.

García, Cybele C, Verónica M Quintana, Viviana Castilla, and Elsa B Damonte. 2018. 'Towards host cell-targeting therapies to treat dengue virus infections', Front. AntiInfect. Drug Discov, 7: 45.

Ghidan, Alaa Y, Tawfiq M Al-Antary, Akl M Awwad, Osama Y Ghidan, Salah-Eddin A Araj, and Mazen A Ateyyat. 2018. 'Comparison of different green synthesized nanomaterials on green peach aphid as aphicidal potential', FEB-FRESENIUS ENVIRONMENTAL BULLETIN: 7009.

Ghidan, A. Y., Al-Antary, T. M., Awwad, A. M., \& Akash, M. W. (2006). APHIDICIDAL POTENTIAL OF GREEN SYNTHESIZED MAGNESIUM HYDROXIDE NANOPARTICLES USING Olea europaea LEAVES EXTRACT. ARPN Journal of Agricultural and Biological Science. VOL. 12, NO. 10.

Ghidan, A. Y., Al-Antary, T. M., Salem, N. M., \& Awwad, A. M. (2017). Facile green synthetic route to the zinc oxide (ZnONPs) nanoparticles: effect on green peach aphid and antibacterial activity. Journal of Agricultural Science, 9(2), 131.

González de Requena, Daniel, Francisco Blanco, Teresa Garcia-Benayas, Inmaculada Jiménez-Nácher, Juan González-Lahoz, and Vincent Soriano. 2003. 'Correlation between lopinavir plasma levels and lipid abnormalities in patients taking lopinavir/ritonavir', AIDS patient care and STDs, 17: 443-45.

Gorbalenya, Alexander E, Susan C Baker, Ralph Baric, Raoul J de Groot, Christian Drosten, Anastasia A Gulyaeva, Bart L Haagmans, Chris Lauber, Andrey M Leontovich, and Benjamin W Neuman. 2020. 'Severe acute respiratory syndrome-related coronavirus: The species and its viruses-a statement of the Coronavirus Study Group'.

Greish, Khaled, Aanchal Mathur, Moiz Bakhiet, and Sebastien Taurin. 2018. 'Nanomedicine: is it lost in translation?', Therapeutic delivery, 9: 269-85.

Guo, Minglan, Jingguang Wei, Xiaohong Huang, Youhua Huang, and Qiwei Qin. 2012. 'Anti-viral effects of $\beta$ defensin derived from orange-spotted grouper (Epinephelus coioides)', Fish \& shellfish immunology, 32: 828-38.

Haddadin, MSY. 2010. 'Effect of olive leaf extracts on the growth and metabolism of two probiotic bacteria of intestinal origin', Pakistan Journal of Nutrition, 9: 78793. 
$\mathrm{Hu}$, Tony Y, Matthew Frieman, and Joy Wolfram. 2020. 'Insights from nanomedicine into chloroquine efficacy against COVID-19', Nature Nanotechnology, 15: 24749.

Huang, Chaolin, Yeming Wang, Xingwang Li, Lili Ren, Jianping Zhao, Yi Hu, Li Zhang, Guohui Fan, Jiuyang $\mathrm{Xu}$, and Xiaoying Gu. 2020. 'Clinical features of patients infected with 2019 novel coronavirus in Wuhan, China', The Lancet, 395: 497-506.

Huang, Youhua, Xiaohong Huang, Jia Cai, Zhengliang OuYang, Shina Wei, Jingguang Wei, and Qiwei Qin. 2015. 'Identification of orange-spotted grouper (Epinephelus coioides) interferon regulatory factor 3 involved in anti-viral immune response against fish RNA virus', Fish \& shellfish immunology, 42: 345-52.

Jefferson, Tom, Vittorio Demicheli, Jon Deeks, and Daniela Rivetti. 2002. 'Amantadine and rimantadine for influenza A in adults', Cochrane Database of Systematic Reviews.

Jin, Ying-Hui, Lin Cai, Zhen-Shun Cheng, Hong Cheng, Tong Deng, Yi-Pin Fan, Cheng Fang, Di Huang, Lu-Qi Huang, and Qiao Huang. 2020. 'A rapid advice guideline for the diagnosis and treatment of 2019 novel coronavirus (2019-nCoV) infected pneumonia (standard version)', Military Medical Research, 7: 4.

Kalsum, Nurbani, Ahmad Sulaeman, Budi Setiawan, and IWT Wibawan. 2017. 'Preliminary studies of the immunomodulator effect of the propolis Trigona spp. extract in a mouse model', IOSR Journal of Agriculture and Veterinary Science, 10: 75-80.

Kapoor, Krishan Mohan, and Aanandita Kapoor. 2020. 'Role of Chloroquine and Hydroxychloroquine in the Treatment of COVID-19 Infection-A Systematic Literature Review', Medrxiv.

Kingsbury, Joanne, Rob Lake, Joanne Hewitt, Erasmus Smit, and Nicola King. 2020. 'POTENTIAL FOR FOODBORNE TRANSMISSION OF COVID-19: LITERATURE REVIEW UPDATE', Update.

Krishna, Sanjeev, and Nicholas J White. 1996. 'Pharmacokinetics of quinine, chloroquine and amodiaquine', Clinical pharmacokinetics, 30: 263-99.

Krogstad, Donald J, Ilya Y Gluzman, Dennis E Kyle, AM Oduola, Samuel K Martin, Wilbur K Milhous, and Paul H Schlesinger. 1987. 'Efflux of chloroquine from Plasmodium falciparum: mechanism of chloroquine resistance', Science, 238: 1283-85.

Lai, Chih-Cheng, Tzu-Ping Shih, Wen-Chien Ko, Hung-Jen Tang, and Po-Ren Hsueh. 2020. 'Severe acute respiratory syndrome coronavirus 2 (SARS-CoV-2) and corona virus disease-2019 (COVID-19): the epidemic and the challenges', International journal of antimicrobial agents: 105924 .

Lee, Ok-Hwan, Boo-Yong Lee, Junsoo Lee, Hee-Bong Lee, Jong-Youn Son, Cheon-Seok Park, Kalidas Shetty, and Young-Cheul Kim. 2009. 'Assessment of phenolicsenriched extract and fractions of olive leaves and their antioxidant activities', Bioresource technology, 100: 6107-13.
Li, Liu-Cheng, and Lian-Di Kan. 2017. 'Traditional Chinese medicine for pulmonary fibrosis therapy: progress and future prospects', Journal of ethnopharmacology, 198: 45-63.

Liu, Cynthia, Qiongqiong Zhou, Yingzhu Li, Linda V Garner, Steve P Watkins, Linda J Carter, Jeffrey Smoot, Anne C Gregg, Angela D Daniels, and Susan Jervey. 2020. "Research and development on therapeutic agents and vaccines for COVID-19 and related human coronavirus diseases." In.: ACS Publications.

Liu, Wenshe, Jared S Morse, Tyler Lalonde, and Shiqing $\mathrm{Xu}$. 2020. 'Learning from the past: possible urgent prevention and treatment options for severe acute respiratory infections caused by 2019-nCoV', Chembiochem.

Lu, Hongzhou. 2020. 'Drug treatment options for the 2019new coronavirus (2019-nCoV)', Bioscience trends, 14: 69-71.

Mariadoss, A. V. A., Ramachandran, V., Shalini, V., Agilan, B., Franklin, J. H., Sanjay, K., Ghidan, A. Y. Alantary, T. M... \& Ernest, D. (2019). Green synthesis, characterization and antibacterial activity of silver nanoparticles by Malus domestica and its cytotoxic effect on (MCF-7) cell line. Microbial pathogenesis, $135,103609$.

Marzouk, Brahim. 2013. 'Characterization of bioactive compounds in Tunisian bitter orange (Citrus aurantium L.) peel and juice and determination of their antioxidant activities', BioMed research international, 2013.

McNamara, Tracey, Juergen A Richt, and Larry Glickman. 2020. 'A critical needs assessment for research in companion animals and livestock following the pandemic of COVID-19 in humans', Vector-Borne and Zoonotic Diseases, 20: 393-405.

Nicastri, Emanuele, Nicola Petrosillo, Tommaso Ascoli Bartoli, Luciana Lepore, Annalisa Mondi, Fabrizio Palmieri, Gianpiero D'Offizi, Luisa Marchioni, Silvia Murachelli, and Giuseppe Ippolito. 2020. 'National institute for the infectious diseases "L. Spallanzani", IRCCS. Recommendations for COVID-19 clinical management', Infectious Disease Reports, 12.

Omar, Syed Haris. 2010. 'Cardioprotective and neuroprotective roles of oleuropein in olive', Saudi Pharmaceutical Journal, 18: 111-21.

Owen, RW, R Haubner, Gl Würtele, WE Hull, Bartsh Spiegelhalder, and H Bartsch. 2004. 'Olives and olive oil in cancer prevention', European Journal of Cancer Prevention, 13: 319-26.

Oyetayo, VO, and FL Oyetayo. 2005. 'Potential of probiotics as biotherapeutic agents targeting the innate immune system', African Journal of biotechnology, 4: 123-27.

Paraguassu, Eber Coelho, Huijun Chen, Fei Zhou, Zhe Xu, and Meiyun Wang. 2020. 'Coronavirus and COVID-19: The latest news and views from the scientific community about the new coronavirus and COVID-19', Brazilian Journal of Implantology and Health Sciences, 2: 96-109.

Pereira, Ana Paula, Isabel CFR Ferreira, Filipa Marcelino, 
Patricia Valentão, Paula B Andrade, Rosa Seabra, Leticia Estevinho, Albino Bento, and José Alberto Pereira. 2007. 'Phenolic compounds and antimicrobial activity of olive (Olea europaea L. Cv. Cobrançosa) leaves', Molecules, 12: 1153-62.

Philippidis, Alex. 2020. 'COVID-19: Top 60 Drug Treatments in Development: The biopharma industry is ramping up the development of dozens of potential drug therapies and clinical testing in an all-hands effort to combat the pandemic', Genetic Engineering \& Biotechnology News, 40: 10-13.

Prabakaran, Ponraj, Xiaodong Xiao, and Dimiter S Dimitrov. 2004. 'A model of the ACE2 structure and function as a SARS-CoV receptor', Biochemical and biophysical research communications, 314: 235-41.

Pradhan, Deepak, Prativa Biswasroy, Goutam Ghosh, and Goutam Rath. 2020. 'A Review of Current Interventions for COVID-19 Prevention', Archives of Medical Research.

Ratner, Daniel, and Mark A Ratner. 2004. Nanotechnology and homeland security: New weapons for new wars (Prentice Hall Professional).

Roberfroid, Marcel B. 2000. 'Prebiotics and probiotics: are they functional foods?', The American journal of clinical nutrition, 71: 1682S-87S.

Rolain, Jean-Marc, Philippe Colson, and Didier Raoult. 2007. 'Recycling of chloroquine and its hydroxyl analogue to face bacterial, fungal and viral infections in the 21 st century', International journal of antimicrobial agents, 30: 297-308.

Ruiz-Gutiérrez, Valentina, Alonso Pérez-Espinosa, Carmen María Vázquez, and Consuelo Santa-Maria. 1999. 'Effects of dietary fats (fish, olive and high-oleic-acid sunflower oils) on lipid composition and antioxidant enzymes in rat liver', British Journal of Nutrition, 82: 233-41.

Ramachandran, V., Arokia Vijaya Anand, M., David, E., Venkatachalam, K., Vijayakumar, S., Sankaran, V., ... \& Ghidan, A. (2020). Antidiabetic Activity of Gold Nanoparticles Synthesized Using Wedelolactone in RIN-5F Cell Line. Antioxidants, 9(1), 8.

Ryan, Danielle, Kevin Robards, and Shimon Lavee. 1999. 'Determination of phenolic compounds in olives by reversed-phase chromatography and mass spectrometry', Journal of Chromatography A, 832: 87-96.

Sak, Katrin. 2012. 'Chemotherapy and dietary phytochemical agents', Chemotherapy research and practice, 2012.

Salata, Cristiano, Arianna Calistri, Cristina Parolin, Aldo Baritussio, and Giorgio Palù. 2017. 'Anti-viral activity of cationic amphiphilic drugs', Expert review of antiinfective therapy, 15: 483-92.

SHIQIN, HOWE. 2015. 'VIRAL RESPIRATORY INFECTIONS IN SINGAPORE'.

Staničova, J, P Miškovsky, and V Šutiak. 2001. 'Amantadine: an antiviral and antiparkinsonian agent', Vet. Med. Czech, 46: 244-56.

Sun, Duxin. 2020. 'Remdesivir with IV Administration
Alone is Unlikely to Achieve Adequate Efficacy and Pulmonary Delivery should be Investigated in COVID19 Patients', Ann Arbor, 1001: 48109.

Tominack, RL, and FG Hayden. 1987. 'Rimantadine hydrochloride and amantadine hydrochloride use in influenza A virus infections', Infectious disease clinics of North America, 1: 459-78.

Trape, Jean-François, Gilles Pison, Marie-Pierre Preziosi, Catherine Enel, Annabel Desgrées du Loû, Valérie Delaunay, Badara Samb, Emmanuel Lagarde, JeanFrançois Molez, and François Simondon. 1998. 'Impact of chloroquine resistance on malaria mortality', Comptes Rendus de l'Académie des Sciences-Series IIISciences de la Vie, 321: 689-97.

Tuck, Kellie L, and Peter J Hayball. 2002. 'Major phenolic compounds in olive oil: metabolism and health effects', The Journal of nutritional biochemistry, 13: 636-44.

Vanderhaeghe, Lorna R. 2000. The Immune System Cure: Optimize Your Immune System in 30 Days-The Natural Way! (Kensington Books).

Velavan, Thirumalaisamy P, and Christian G Meyer. 2020. 'The COVID-19 epidemic', Tropical Medicine \& international health, 25: 278.

Visioli, Francesco, Claudio Galli, Francis Bornet, Alissa Mattei, Rossana Patelli, Giovanni Galli, and Donatella Caruso. 2000. 'Olive oil phenolics are dose-dependently absorbed in humans', FEBS letters, 468: 159-60.

Vissers, Maud N, Peter L Zock, Annet JC Roodenburg, Rianne Leenen, and Martijn B Katan. 2002. 'Olive oil phenols are absorbed in humans', The Journal of nutrition, 132: 409-17.

Wang, Manli, Ruiyuan Cao, Leike Zhang, Xinglou Yang, Jia Liu, Mingyue Xu, Zhengli Shi, Zhihong Hu, Wu Zhong, and Gengfu Xiao. 2020. 'Remdesivir and chloroquine effectively inhibit the recently emerged novel coronavirus (2019-nCoV) in vitro', Cell research, 30: 269-71.

Weber, Norbert D, Douglas O Andersen, James A North, Byron K Murray, Larry D Lawson, and Bronwyn G Hughes. 1992. 'In vitro virucidal effects of Allium sativum (garlic) extract and compounds', Planta medica, 58: 417-23.

Wellems, Thomas E, and Christopher V Plowe. 2001. 'Chloroquine-resistant malaria', The Journal of infectious diseases, 184: 770-76.

Wimmer, Eckard, Steffen Mueller, Terrence M Tumpey, and Jeffery K Taubenberger. 2009. 'Synthetic viruses: a new opportunity to understand and prevent viral disease', Nature biotechnology, 27: 1163.

Wu, Jian, Jun Liu, Xinguo Zhao, Chengyuan Liu, Wei Wang, Dawei Wang, Wei Xu, Chunyu Zhang, Jiong Yu, and Bin Jiang. 2020. 'Clinical characteristics of imported cases of COVID-19 in Jiangsu province: a multicenter descriptive study', Clin Infect Dis, 10.

Yang, Xiaobo, Yuan Yu, Jiqian Xu, Huaqing Shu, Hong Liu, Yongran $\mathrm{Wu}$, Lu Zhang, Zhui Yu, Minghao Fang, and Ting Yu. 2020. 'Clinical course and outcomes of critically ill patients with SARS-CoV-2 pneumonia in 
Wuhan, China: a single-centered, retrospective, observational study', The Lancet Respiratory Medicine.

Zhang, Wen, Yan Zhao, Fengchun Zhang, Qian Wang, Taisheng Li, Zhengyin Liu, Jinglan Wang, Yan Qin, Xuan Zhang, and Xiaowei Yan. 2020. 'The use of antiinflammatory drugs in the treatment of people with severe coronavirus disease 2019 (COVID-19): The experience of clinical immunologists from China', Clinical Immunology: 108393.

Zhu, Na, Dingyu Zhang, Wenling Wang, Xingwang Li, Bo Yang, Jingdong Song, Xiang Zhao, Baoying Huang, Weifeng Shi, and Roujian Lu. 2020. 'A novel coronavirus from patients with pneumonia in China, 2019', New England Journal of Medicine. 